\title{
Endoluminal Stenting for Unprotected Left Main Stenosis in Takayasu's Arteritis
}

\author{
Kirti Punamiya, MD, Eric R. Bates, ${ }^{*}$ MD, Michael J. Shea, MD, and David W.M. Muller, MBBS \\ We describe 2 patients with Takayasu's arteritis and left main coronary stenosis who were \\ considered poor candidates for surgical revascularization. Elective left main coronary \\ artery balloon angioplasty followed by endoluminal stenting was performed with excellent \\ results. At 3-mo follow-up, one patient had evidence of in-stent restenosis, but the other \\ remained asymptomatic. In selected patients with Takayasu's arteritis and left main \\ coronary stenosis, elective endoluminal stenting can be used as a definitive procedure or \\ as a bridge to surgical revascularization. Cathet. Cardiovasc. Diagn. 40:272-275, \\ 1997. 1997 Wiley-Liss, Inc.
}

Key words: coronary stenting; Takayasu's arteritis; left main stenosis

\section{INTRODUCTION}

Takayasu's arteritis is a chronic inflammatory disease primarily affecting large arteries, and it occurs most frequently in females in their reproductive years [1-3]. There are reports of Takayasu's arteritis presenting as acute coronary ischemic syndromes [4-5], but the overall incidence of coronary involvement is low and when it does occur, there is usually involvement of aortoostial segments and the proximal coronary arteries [6,7]. Percutaneous transluminal coronary angioplasty (PTCA) of the native coronary arteries in Takayasu's disease has been reported [5]. In this report we describe, for the first time, 2 adult patients with Takayasu's arteritis who underwent elective endoluminal stenting of the left main coronary artery.

\section{CASE REPORTS}

\section{Case 1}

A 39-year-old woman with a history of tobacco use, hypertension, and Takayasu's arteritis presented to an outside hospital with shortness of breath and atypical chest pain. Cardiac enzymes and the initial electrocardiogram were not diagnostic of myocardial infarction. Her initial workup revealed a dilated ascending and descending aorta with moderate aortic regurgitation, bilateral subclavian artery occlusion, and the suggestion of a limited aortic dissection distal to the origin of the left subclavian artery. Selective coronary arteriography showed a right dominant coronary circulation with an angiographically normal right coronary artery. The left main coronary artery (LM) had a tight stenosis distally, which extended into the bifurcation involving the ostia of the left anterior descending artery (LAD) and the left circumflex artery (LCX), as shown in Figure 1. The distal LAD received collaterals from the right coronary artery. She was referred to our institution for aortic valve replacement and bypass graft surgery. Physical examination revealed a thin woman with lower-extremity blood pressure 140/90 $\mathrm{mm} \mathrm{Hg}$, a regular pulse at $64 \mathrm{bpm}$, normal S1, soft S2, audible $\mathrm{S} 3$, and a grade $2 / 6$ aortic regurgitation murmur. Upper-extremity pulses were absent on the left side and faint on the right. Electrocardiograms were not suggestive of ischemia. A spiral computerized tomographic scan and magnetic resonance imaging excluded an aortic dissection. After this initial workup, the patient developed further chest pain with shortness of breath and rapid progression to pulmonary edema. This required mechanical ventilation over the next 10 days. Her EKG at this time revealed an evolving anteroseptal myocardial infarction with elevation of cardiac enzymes. She had a slow recovery, which was complicated by thrombophlebitis and occlusion of the left subclavian, right internal jugular, and right iliac veins. Laboratory findings were significant for mildly elevated sedimentation rate (ESR 31). Antinuclear antibody, anti-DNA antibody, and anti-phospholipid antibody were negative. Because coronary bypass surgery with aortic valve replacement was considered an unfavorable option in view of the poor arterial and venous conduits and the recent myocardial infarction, the patient was referred for an elective percutaneous coronary intervention.

From the Department of Internal Medicine, Division of Cardiology, University of Michigan Medical Center, Ann Arbor, Michigan

*Correspondence to Eric Bates, M.D., B1F245 University Hospital, 1500 East Medical Center Drive, Ann Arbor, MI 48109-0022.

Received 26 June 1996; Revision accepted 15 October 1996 


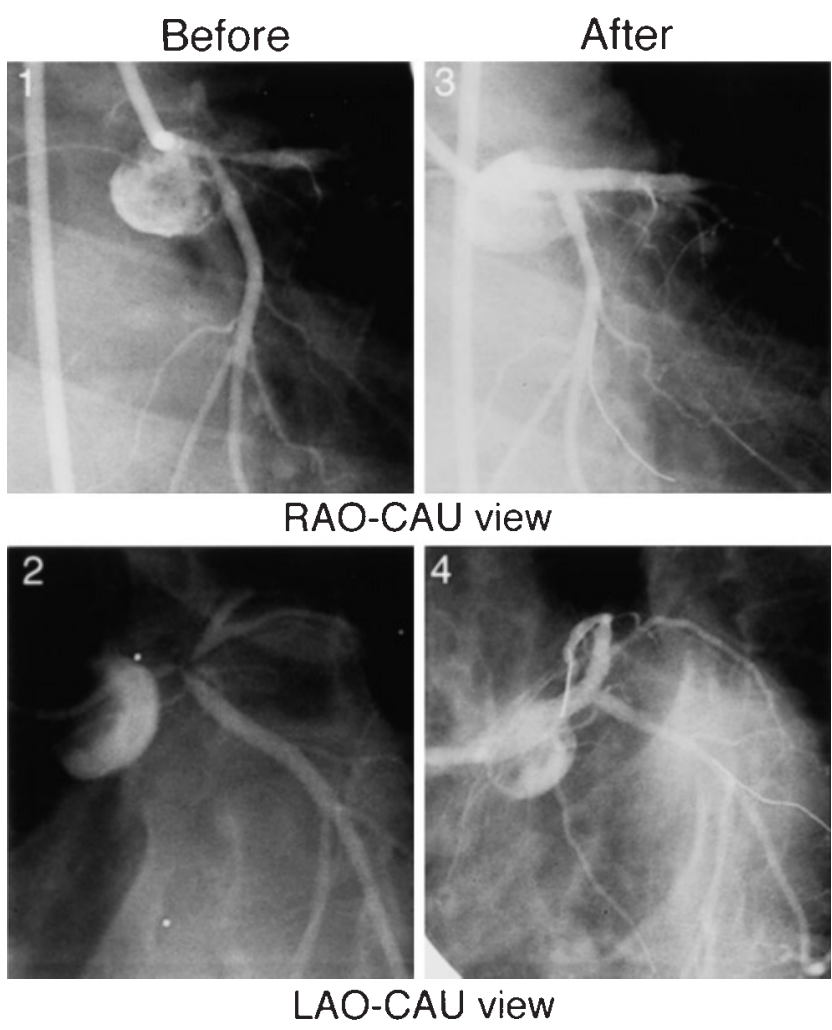

Fig. 1. Patient 1. 1,2: RAO-caudal (RAO-CAU) and LAO-caudal (LAO-CAU) projections of the LCA, showing LM stenosis with involvement of the LAD and LCX ostia. 3,4: Similar projections after stent deployment in the ostia of the LAD, LCX, and LM, showing $0 \%$ residual stenosis.

A 9F JL 4 Brite Tip guide catheter (Cordis Corp., Miami Lakes, FL) was used to engage the left coronary artery. After placing an exchange length 0.014" HiTorque Floppy II Extra Support wire (Advanced Cardiovascular Systems, Santa Clara, CA) in both the LAD and the LCX artery, a 3.0-mm Lifestream balloon (Advanced Cardiovascular Systems) was used to predilate the LAD ostial stenosis. A 7-mm half of a 3.5-mm Palmaz-Schatz coronary stent (Johnson \& Johnson Interventional Systems, Warren, NJ) was placed in the ostium of the LAD (Fig. 2). The second 7-mm half of the 3.5-mm stent was placed in the ostium of the LCX artery (Fig. 2). The LM artery was then stented using a $10-\mathrm{mm}$ nonarticulated Palmaz biliary stent (Johnson \& Johnson Interventional Systems). The LM and LAD stents were postdilated using a 4.0-mm NC Bandit balloon (SciMed, Maple Grove, $\mathrm{MN})$ at $16 \mathrm{~atm}$ pressure. The circumflex artery stent was postdilated using a $3.25-\mathrm{mm}$ NC Bandit balloon. There was no residual stenosis, and flow was excellent (Figs. 1, 2 ) in both the LAD and LCX arteries. The patient was maintained on ticlopidine for 6 weeks and aspirin indefinitely, in addition to prednisone and cyclophosphamide. She was also started on warfarin $1 \mathrm{wk}$ after the stent implantation as a prophylaxis against future deep venous thrombosis.

The patient presented 13 weeks later with a short history of recurrent chest discomfort. Repeat angiography demonstrated a $90 \%$ stenosis at the ostium of the LAD in the small gap between the left main and LAD stents, and a 90\% in-stent restenosis of the proximal LCX artery (Fig. $3)$. These lesions were successfully treated with PTCA, reducing the stenoses to $<20 \%$.

\section{Case 2}

This 34-year-old woman first presented in 1994 with chest tightness. Angiography revealed bilateral subclavian artery occlusion and carotid artery stenoses, narrowing of the distal aorta below the diaphragm, and $90 \%$ stenosis at the ostium of the left main (LM) coronary artery (Fig. 4). A diagnosis of Takayasu's arteritis was made, and she was treated with prednisone and cyclophosphamide with some improvement. In the ensuing months, she developed a non-Q-wave myocardial infarction secondary to a hypertensive crisis. In January 1996, she presented with vague symptoms and changes in mental status. Clinical examination revealed an alert patient in no distress with a systemic blood pressure of $150 / 82 \mathrm{~mm} \mathrm{Hg}$, pulse $100 \mathrm{bpm}$ and regular, and 24 respirations per min. Cardiovascular examination was significant for mild mitral regurgitation and moderate to severe pulmonary hypertension. The patient had a minimal residual rightsided neurological motor deficit from the previous stroke. Upper-extremity pulses were absent, and lower-extremity pulses were bounding. There was T-wave inversion in the anterior and lateral EKG leads. C-reactive proteins were negative, and sedimentation rate was normal. Antinuclear antibody, anti-DNA antibody, and anti-phospholipid antibody were negative. This hospitalization was complicated by an asystolic cardiac arrest requiring 10 min of cardiopulmonary resuscitation and by right hemiparesis due to a parietooccipital infarct. Initial echocardiography after the cardiac arrest revealed an ejection fraction of $20 \%$ with marked anterolateral hypokinesia. Repeat echocardiography revealed normalization of left ventricular function consistent with recovery of stunned myocardium. Persantine thallium imaging demonstrated a large reversible defect in the anterolateral distribution. Coronary bypass surgery was not considered a favorable option because of the recent stroke, active vasculitis, and fear that the aortic anastomotic sites of the vein grafts would stenose. She was referred for a percutaneous intervention.

Repeat coronary angiography in February 1996 confirmed the ostial location of the left main coronary stenosis (Fig. 4). The distal half of the normal left anterior descending artery received collaterals from the right coronary artery. Balloon angioplasty and elective stenting 

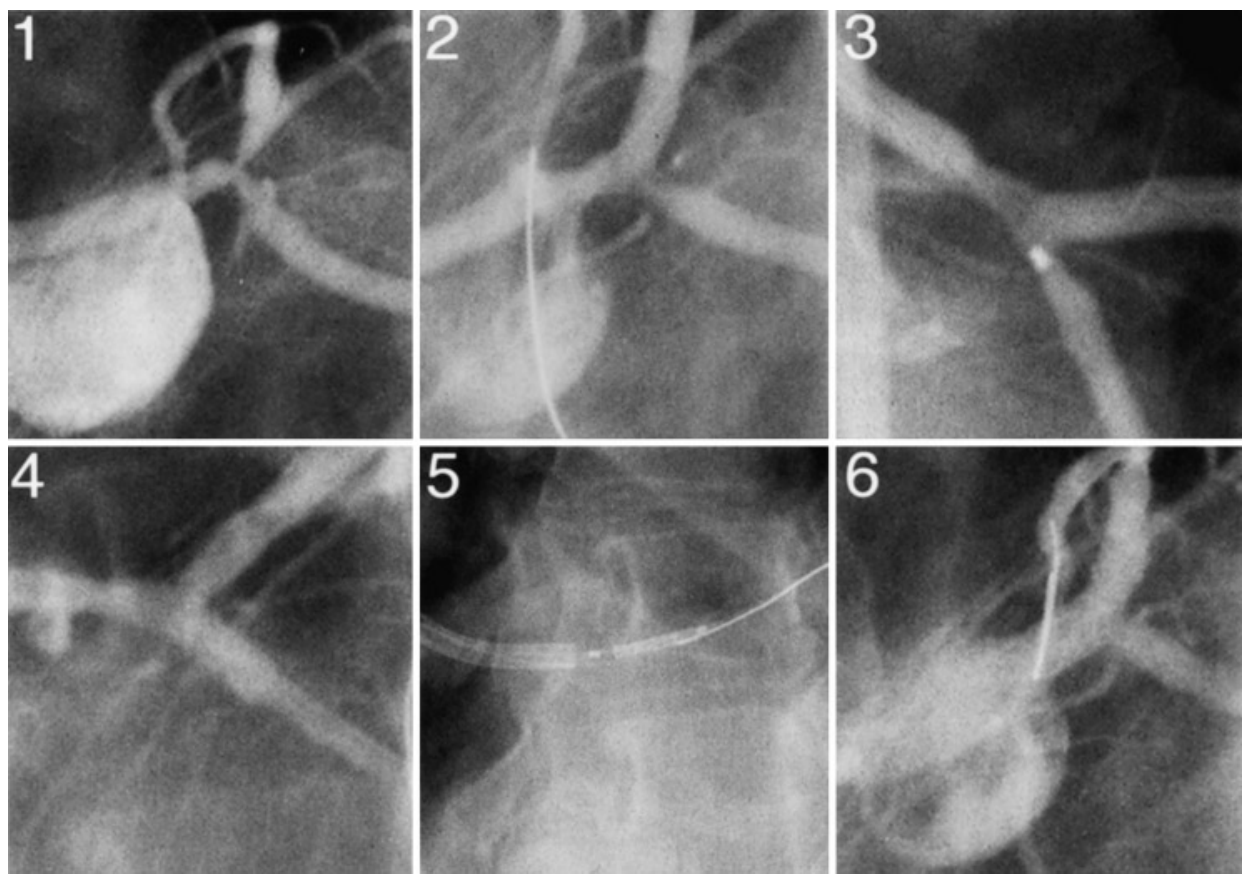

Fig. 2. Patient 1. LAO-caudal projection, showing (1) baseline anatomy, (2) poststent placement in the ostium of the LAD, (3) positioning the stent in the LCX ostium (note alignment technique, using balloon marker as guide for stent positioning), (4) poststent placement in LCX ostium, (5) positioning the 10-mm Palmaz biliary stent in the left main artery, and (6) final angiography with minimal residual narrowing at the bifurcation site.
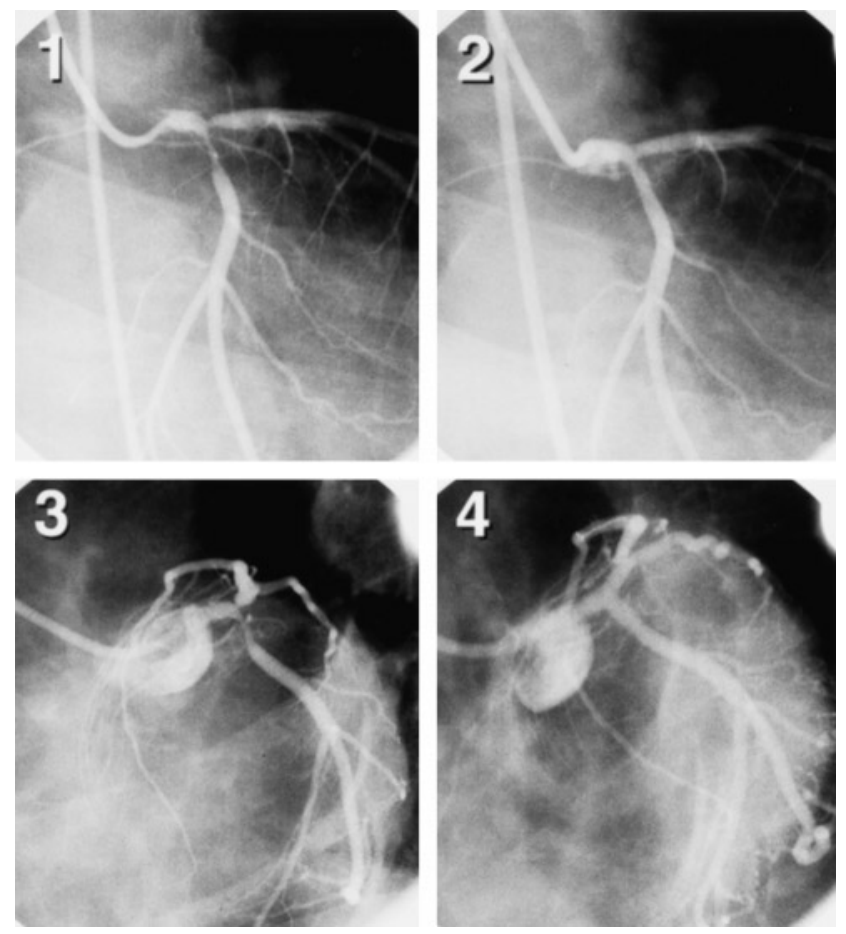

Fig. 3. Patient 1. RAO (top) and LAO (bottom) projections before (left) and after (right) PTCA for restenosis. of the left main coronary artery were successfully performed using a 9F JL 4.0 Brite Tip guiding catheter with sideholes (Cordis Corp.). After seating the catheter safely at the os of the left coronary artery, an exchange length 0.014" Hi-Torque Floppy II Extra Support wire (Advanced Cardiovascular Systems) was passed distal to the stenosis into the left anterior descending artery. An initial balloon inflation was performed using a 3.5-mm Lifestream balloon (Advanced Cardiovascular Systems) at 6 atm. A $15-\mathrm{mm}$ nonarticulated Palmaz biliary stent (Johnson \& Johnson Interventional Systems) was deployed in the ostial segment of the left main, using the Lifestream balloon. The stent was postdilated using a 4.0-mm NC Cobra balloon (SciMed) at $18 \mathrm{~atm}$. Final angiography revealed $0 \%$ residual stenosis and good distal flow (Fig. 3). Besides prednisone and cytoxan, the patient was maintained on ticlopidine for $6 \mathrm{wk}$ and on aspirin indefinitely. This patient remained asymptomatic at 3-mo follow-up.

\section{DISCUSSION}

Involvement of the coronary arteries in Takayasu's arteritis has been well-described by various investigators from autopsy and clinical data [5-8]. Treatment strategies to improve clinical outcomes have ranged from medical therapy with steroids and immunosuppressive agents [9] 

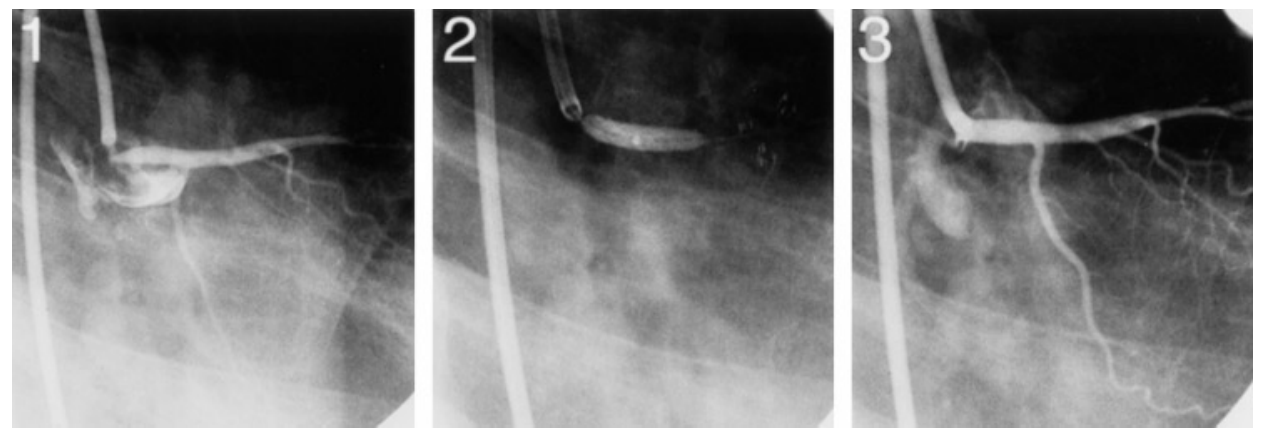

Fig. 4. Patient 2. 1: Baseline RAO projection showing ostial LM stenosis. 2: Deployment of 15-mm nonarticulated Palmaz biliary stent in the LM position. 3: Final result poststenting, with $0 \%$ residual stenosis.

to coronary balloon dilatation [5]. Antelmi et al. [5] reported that PTCA for Takayasu's arteritis was limited by high-grade residual stenosis presumably due to recoil. Ozdil et al. [10] reported endoluminal stenting of an ostial lesion in a mesenteric artery in Takayasu's arteritis. To our knowledge, there is no published literature on the use of endoluminal coronary stenting as an adjunct to PTCA in Takayasu's arteritis.

Previous studies have shown an increased risk of failure of surgical revascularization during active disease [11]. Findings of histologically active arteritis in this group correlate poorly with clinical assessment of activity [12]. Our patients were not suitable for cardiac surgical revascularization because of active disease, recent myocardial infarction, or cerebrovascular event, and fear of low graft patency rates in a diseased aorta.

PTCA for atheromatous left main stenosis has been reported but is associated with suboptimal short-term and long-term results because of immediate elastic recoil and a high incidence of restenosis [13]. Percutaneous angioplasty has been attempted in coronary and renal arteries with Takayasu's arteritis, and is also associated with high-grade residual stenosis [5,14]. The outcomes of our 2 patients suggest that elective stenting can be performed in ostial and distal left main stenoses, especially when surgery is a poor option. In both cases, angiograms after the initial balloon dilatation revealed significant elastic recoil. With endoluminal stenting, we were able to achieve large minimum luminal diameters without residual stenoses. Both patients had collateral circulation to the LAD artery, which improved the margin of safety of our procedures. Neither patient required intraaortic balloon counterpulsation or cardiopulmonary bypass support.

One of the patients had restenosis in the ostium of the LAD with in-stent restenosis of the LCX artery. This was successfully treated by PTCA with an excellent angiographic result. It is likely, however, that active arteritis, particularly in the presence of a bifurcation lesion of the left main coronary artery, is associated with a high restenosis rate. Nonetheless, we believe that endoluminal stenting of the left main coronary artery is an option for patients with Takayasu's arteritis who are not good candidates for surgical revascularization.

\section{REFERENCES}

1. Nakao K, Ikeda M, Kimata S, Niitani H, Niyahara M: Takayasu's arteritis. Clinical report of eighty-four cases and immunological studies of seven cases. Circulation 35:1141-1155, 1967.

2. Subramanyam R, Joy J, Balakrishna KG: Natural history of aortoarteritis (Takayasu's disease). Circulation. 80:429-437, 1989.

3. Sen P: "Aorto Arteritis: A Monograph Based on a Study of 101 Cases." Bombay: Tat McGraw Hill, 1973.

4. Trazzera S, Colasacco J, Ong L: Takayasu's arteritis with unstable angina and aortic insufficiency. Am Heart J 130:1122-1124, 1995.

5. Antelmi I, Magalhaes L, Caramelli B, Nakano O, Tranchesi B Jr, Nicolau J, Noogueira P, Machado N, Ballotti G, Pileggi F: Rescue PTCA in a 16 year old boy with Takayasu's disease and evolving myocardial infarction. Arq Bras Cardiol 60:37-38, 1993.

6. Kerr G, Hallahan C, Goirdano J, Leavitt R, Fauci A, Rottem M, Hoffman G: Takayasu's arteritis. Ann Intern Med 120:919-929, 1994.

7. Cipriano PR, Silverman JF, Perlroth MG, Griepp RB, Wexler L: Coronary arterial narrowing in Takayasu's aortitis. Am J Cardiol 39:744-750, 1977.

8. Rose AG, Sinclair-Smith CC: Takayasu's arteritis-A study of 16 autopsy cases. Arch Pathol Lab Med 104:231-237, 1980.

9. Shelhamer JH, Volkman DJ, Parillo JE, Lawley TJ, Johnston MR, Fauci AS: Takayasu's arteritis and its therapy. Ann Intern Med 103:121-126, 1985.

10. Ozdil E, Krajcer Z, Angelini P: Percutaneous balloon angioplasty with adjunctive stent placement in the mesentric vessels in a patient with Takayasu's arteritis. Circulation 93:1940-1941, 1996.

11. Pajari R, Hekali P, Harjola PT: Treatment of Takayasu's arteritis: An analysis of 29 operated patients. Thorac Cardiovasc Surg 34:176-181, 1986

12. Lagneau P, Michel JB, Vuong PN: Surgical treatment of Takayasu's disease. Ann Surg 205:157-166, 1987.

13. O'Keefe JH, Hartzler GO, Rutherford BD, McConaghey DR, Johnson WL, Giorgi LV, Ligon RW: Left main coronary angioplasty: Early and late results of 127 acute and elective procedures. Am J Cardiol 64:144-147, 1989.

14. Sharma S, Saxena A, Talwar KK, Kaul U, Mehta SN, Rajani M: Renal artery stenosis caused by nonspecific arteritis: Results of treatment with percutaneous angioplasty. AJR 158:417-422, 1992. 